\title{
Discrete surface solitons in two-dimensional anisotropic photonic lattices
}

\author{
Rodrigo A. Vicencio and Sergej Flach \\ Max-Planck-Institut für Physik Komplexer Systeme, Nöthnitzer Str. 38, Dresden 01187, Germany \\ Mario I. Molina \\ Departmento de Física, Facultad de Ciencias, Universidad de Chile, Casilla 653, Santiago, Chile \\ Yuri S. Kivshar \\ Nonlinear Physics Center, Research School of Physical Sciences and Engineering, \\ Australian National University, Canberra ACT 0200, Australia
}

\begin{abstract}
We study nonlinear surface modes in two-dimensional anisotropic periodic photonic lattices and demonstrate that, in a sharp contrast to one-dimensional discrete surface solitons, the mode threshold power is lower at the surface, and two-dimensional discrete solitons can be generated easier near the lattice corners and edges. We analyze the crossover between effectively one- and two-dimensional regimes of the surface-mediated beam localization in the lattice.
\end{abstract}

PACS numbers:

Surface modes have been studied in different branches of physics; in guided wave optics surface states were predicted to exist at interfaces separating periodic and homogeneous dielectric media 1]. The interest in studying surface waves has been renewed recently because the interplay of discreteness and nonlinearity can facilitate the formation of discrete surface solitons [2, 3] at the edge of the waveguide array. That can be understood as the localization of a discrete optical soliton near the surface 4 for powers exceeding a certain threshold value, for which the repulsive effect of the surface is balanced. A similar effect of light localization near the edge of the waveguide array and the formation of surface gap solitons have been predicted and observed for defocusing nonlinear media $[5,6]$.

It is important to analyze how the properties of nonlinear surface waves are modified by the lattice dimensionality, and the first studies of different types of discrete surface solitons in two-dimensional lattices 7, 8, 9, 10 revealed, in particular, that the presence of a surface increases the stability region for two-dimensional (2D) discrete solitons [10] and the threshold power for the edge surface state is slightly higher than that for the corner soliton [9].

In this Letter we consider anisotropic semi-infinite twodimensional photonic lattices and study the crossover between one- and two-dimensional surface solitons emphasizing the crucial effect of the lattice dimensionality on the formation of surface solitons.

We consider a semi-infinite 2D lattice [shown schematically in Fig 2 (a) below], described by the system of coupled-mode equations for the normalized amplitudes $u_{n, m}$ [1, 12],

$$
i \frac{\partial u_{n, m}}{\partial \xi}+\left(V_{n}+V_{m}\right) u_{n, m}+\left|u_{n, m}\right|^{2} u_{n, m}=0
$$

where $\xi$ is the normalized propagation distance. We de-
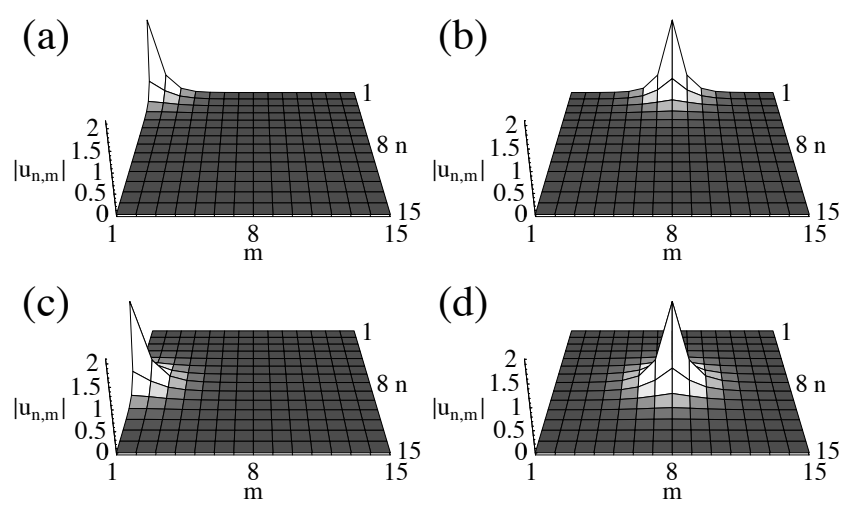

(d)

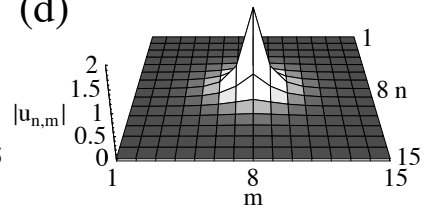

FIG. 1: Examples of (a) corner, (b,c) two edge surface, and (d) central localized nonlinear modes for $\lambda=5$.

fine the lattice coupling as follows:

$$
\begin{array}{r}
V_{n} u_{n, m}=\left\{\begin{array}{lr}
u_{2, m} & n=1, \\
u_{n+1, m}+u_{n-1, m} & n>1,
\end{array}\right. \\
V_{m} u_{n, m}=\alpha\left\{\begin{array}{cc}
u_{n, 2} & n \geq 1, m=1, \\
\left(u_{n, m+1}+u_{n, m-1}\right) & m>1,
\end{array}\right.
\end{array}
$$

where $\alpha$ characterizes the lattice anisotropy.

Linear lattice waves of the form $u_{n, m}(\xi)=$ $u_{0} \sin (k n) \sin (q m) \exp (i \beta \xi)$ satisfy the dispersion relation $\beta_{k q}=2(\cos k+\alpha \cos q)$. In the nonlinear case, we look for localized stationary solutions of the form $u_{n, m}(\xi)=u_{n, m} \exp (i \lambda \xi)$, where the amplitudes $u_{n, m}$ are real, and $\lambda$ is the nonlinear propagation constant. For a given $\lambda$, localized solutions are found in a $15 \times 15$ lattice by using the Newton-Raphson method.

We calculate the power threshold $P_{\text {th }}$ that characterizes the discrete solitons in 2D lattices [13]. We study three different modes: corner [Fig. 1(a)], edge surface [Fig. 1(b,c)] and central [Fig. 1(d)] localized modes. The corner and edge modes represent 2D surface localized 
modes, and the central mode corresponds to a $2 \mathrm{D}$ discrete soliton of an infinite lattice. We find the total power $P=\sum_{n, m}\left|u_{n, m}\right|^{2}$ of all those modes and perform a linear stability analysis [4, 12] for each solution [Fig. 2(a)].

We observe that the threshold power $P_{\text {th }}$ for the surface modes is smaller than the power corresponding to the central mode, with the corner state having the smallest $P_{\mathrm{th}}$. This interesting feature was previously observed for a single nonlinear impurity placed near a boundary of a 2D lattice [14], and it appears also in the anisotropic model. Therefore, in a sharp contrast with one-dimensional (1D) surface solitons, the surface of a $2 \mathrm{D}$ lattice creates an effectively attractive potential for the localized modes that reduces the threshold power for the mode localization.
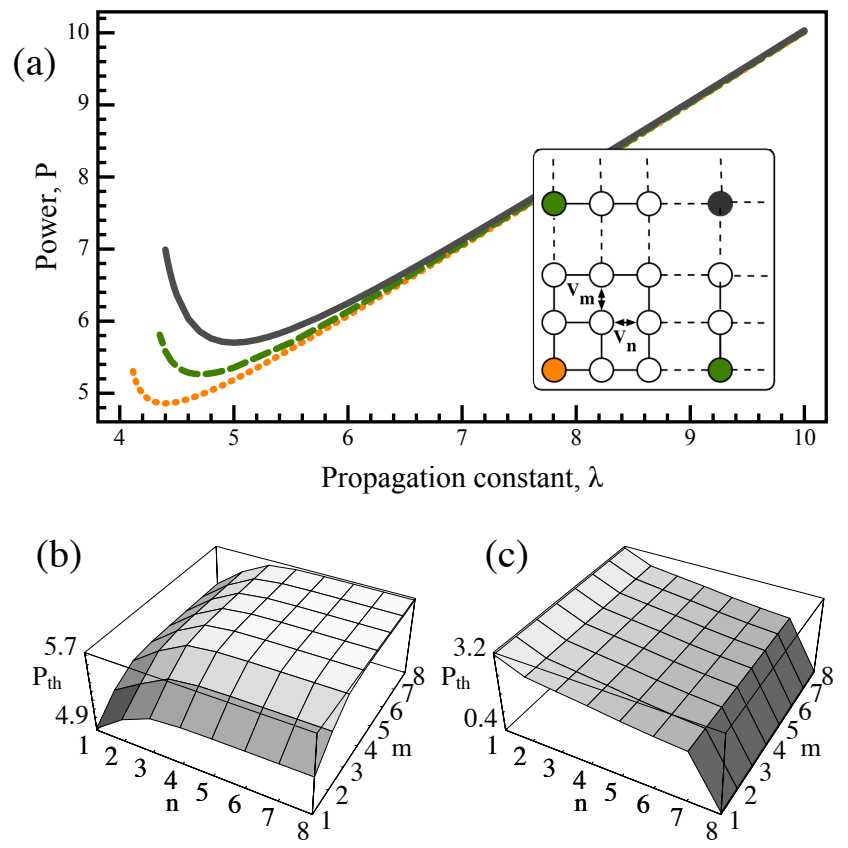

FIG. 2: (Color online) (a) Power diagram of the central (continuous), edges surface (dashed), and corner (dotted) localized modes for the isotropic case $(\alpha=1)$. Inset: Schematic of a semi-infinite two-dimensional lattice. (b,c) Examples of the threshold power landscapes $P_{\mathrm{th}}(n, m)$ for $\alpha=1$ and $\alpha=0$, respectively.

The linear stability analysis of the surface localized modes coincides with the Vakhitov-Kolokolov stability criterion [15]: the surface modes are stable for $d P / d \lambda>$ 0 , and unstable otherwise. To study in more details how the threshold power $P_{\text {th }}$ varies for the modes localized at different points of the lattice, we compute the power threshold landscape: We look numerically for one-peak localized modes centered at different sites of one-quarter of the whole lattice and find the first stable solution at the threshold power which corresponds to $P_{\text {th }}$ at the site $(n, m)$. In Fig. 22(b,c), we show the examples of two landscapes for two different values of the lattice anisotropy $\alpha$. For $\alpha=1, P_{\text {th }}$ grows from the minimum $P_{\text {th }} \approx 4.9$ at the corner $(1,1)$ to two maxima corresponding to the edge surface modes $(8,1)$ and $(1,8)$ and the central mode $(8,8)$. For $\alpha=0$, we observe that the required power to excite a localized mode decreases from the surface $(n=1)$ to the center $(n=8)$. The value $P_{t h}(1, m) \approx 3.2$ corresponds to the threshold power of discrete surface solitons in a semi-infinite array [4]. For 1D localized modes there exists no power threshold in the continuum limit [13], but in our system $P_{\mathrm{th}}(8, m) \approx 0.4$ due to finite-size effects.

Next, we study the effect of the lattice anisotropy on the power threshold of discrete surface solitons. In Fig. 3 we show $P_{\text {th }}$ for different values of the parameter $\alpha(0 \leq$ $\alpha \leq 1$ ), for the four types of localized modes shown in Figs. 11(a-d).

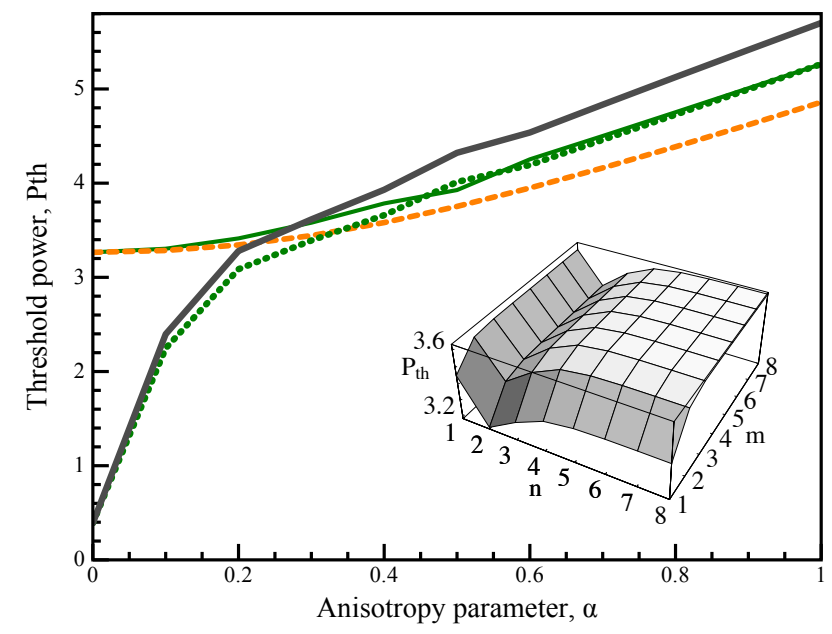

FIG. 3: (Color online) Threshold power $P_{\text {th }}$ vs. $\alpha$ for the central (continuous thick), two edge surface (continuous thin and dotted), and corner (dashed line) localized modes. Inset: $P_{\text {th }}(n, m)$ for $\alpha=0.3$.

When the anisotropy parameter $\alpha$ grows, the threshold power grows for all localized modes. It means that, by increasing the effective dimensionality of the system, the required power to excite a nonlinear discrete localized state also increases. The threshold power for two edge surface modes decreases as $\alpha$ decreases to $\alpha \approx 0.3$, when the power curves cross and diverge. For $\alpha=0$, the edge soliton centered at $(8,1)$ [Fig. 1] c), dotted line in Fig. 3] only interacts with an effective $1 \mathrm{D}$ array $(m=1)$. On the contrary, $P_{\text {th }}$ for the edge surface mode centered at $(1,8)$ [Fig.1(b), continuous-thin line in Fig. 3] approaches the same limit as the corner mode. For $\alpha=0$, this mode only interacts with an effective $1 \mathrm{D}$ chain $(m=8)$ and the surface. An interesting feature of Fig. 3 is that it shows the existence of a critical value of the lattice anisotropy, $\alpha \approx 0.3$ where all powers thresholds almost coincide. In other words, there exists a critical value of the lattice anisotropy where we observe a crossover between 1D and 2D lattices.

To study this interesting effect in more details, we calculate the power threshold landscape $P_{t h}$ for $\alpha=0.3$ [see 
inset in Fig. 3, and observe that the localized mode centered at $(2, m)$ possesses the lowest value of $P_{t h}$ in the array and, in particular, the localized mode centered at $(2,1)$ is the mode with the lowest threshold power. For $\alpha \lesssim 0.3$, the minimum $P_{\text {th }}$ will be located outside of the array boundaries, and intermediate localized states (located between the center and the surfaces of the array) will be the easier states to excite. For 1D semi-infinite arrays [4], it was shown that the power threshold for surface modes decreases as the mode moves away from the surface [see also Fig. 2(c)].
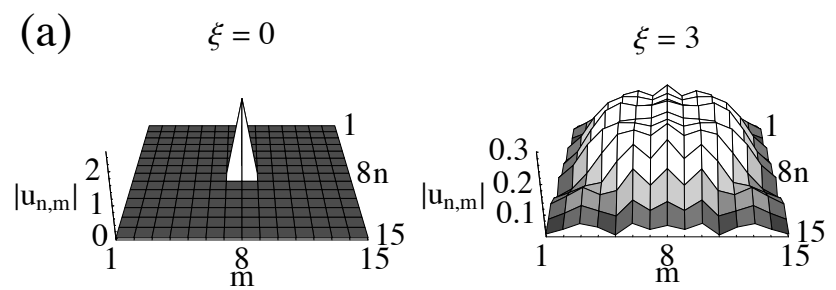

(b)
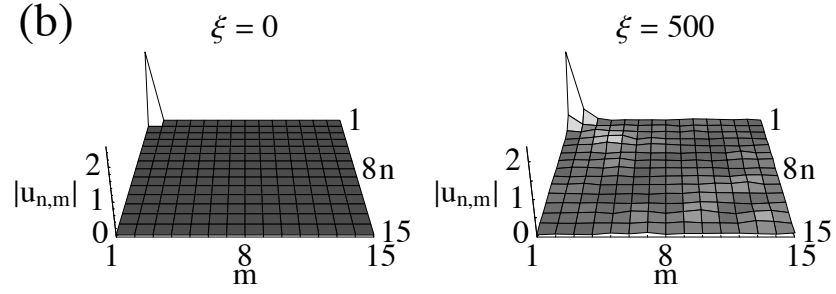

FIG. 4: Generation of surface modes by one-site excitation $(\alpha=1)$ at (a) center and (b) corner of the lattice.

Finally, we study the dynamic generation of localized modes in two-dimensional lattices. We simulate numerically Eqs. (1) by using a one-site excitation $u_{0} \delta_{n, n^{\prime}} \delta_{m, m^{\prime}}$. In Fig. 4 we show our results by plotting the evolution of the mode amplitude $\left|u_{n, m}\right|$ for two different values of $\xi$. As an example, we show the excitation of the corner and central localized modes for the initial amplitude $u_{0}=2.5$. If we try to excite a localized mode at the middle of the lattice for this value of $u_{0}$, this localized state decays quickly, as shown in Fig. 4(a), and the beam diffracts because the input power is below the effective dynamical power threshold. On the contrary, by using the same input power we are able to excite the corner soliton [see Fig. 4(b)] which is dynamically stable in the propagation. It is worth mentioning that, in the one-dimensional limit $(\alpha=0)$ the situation becomes completely different: If we try to excite a surface mode at $n=1$ with the power smaller than a threshold value, but still large enough, the input beam will not decay but will instead move to the neighboring lattice site creating a localized mode there [2].

In conclusion, we have analyzed the properties of discrete surface solitons in two-dimensional anisotropic photonic lattices, and studied the crossover between the oneand two-dimensional regimes of the surface-mediated beam localization in the lattice. In particular, unlike one-dimensional discrete surface solitons, the threshold power of the two-dimensional discrete soliton is lowered by the surface, so that two-dimensional solitons can be generated easier near the lattice corners and edges.

This work was supported by Fondecyt grants 1050193 and 7050173 and the Australian Research Council.
[1] P. Yeh, A. Yariv, and A.Y. Cho, Appl. Phys. Lett. 32, 102 (1978).

[2] K.G. Makris, S. Suntsov, D.N. Christodoulides, G.I. Stegeman, and A. Hache, Opt. Lett. 30, 2466 (2005).

[3] S. Suntsov, K.G. Makris, D.N. Christodoulides, G.I. Stegeman, A. Hache, R. Morandotti, H. Yang, G. Salamo, and M. Sorel, Phys. Rev. Lett. 96, 063901 (2006).

[4] M.I. Molina, R.A. Vicencio, and Yu.S Kivshar, Opt. Lett. 31, 1693 (2006).

[5] Ya.V. Kartashov, V.A. Vysloukh, and L. Torner, Phys. Rev. Lett. 96, 073901 (2006).

[6] C.R. Rosberg, D.N. Neshev, W. Krolikowski, A. Mitchell, R.A. Vicencio, M.I. Molina, and Yu.S. Kivshar, Phys. Rev. Lett. 97, 083901 (2006).

[7] Y.V. Kartashov and L. Torner, Opt. Lett. 31, 2172 (2006)

[8] Y.V. Kartashov, V.A. Vysloukh, D. Mihalache, and L.
Torner, Opt. Lett. 31, 2329 (2006).

[9] K.G. Makris, J. Hudock, D.N. Christodoulides, G.I. Stegeman, O. Manela, and M. Segev, Opt. Lett. 31, 2774 (2006).

[10] H. Susanto, P.G. Kevrekidis, B.A. Malomed, R. Carretero-Gonzalez, and D.J. Franzeskakis, ArXiv:nlin.PS/0607063 (2006).

[11] T. Pertsch, U. Peschel, F. Lederer, J. Burghoff, M. Will, S. Nolte, and A. Tünnermann, Opt. Lett. 29, 468 (2004).

[12] R.A. Vicencio and M. Johansson, Phys. Rev. E 73, 046602 (2006).

[13] S. Flach, K. Kladko, and R.S. MacKay, Phys. Rev. Lett. 78, 1207 (1997).

[14] M.I. Molina, Phys. Rev. B 74, 045412 (2006).

[15] Yu.S. Kivshar and G.P. Agrawal, Optical Solitons: From Fibers to Photonic Crystals (Academic, San Diego, 2003). 